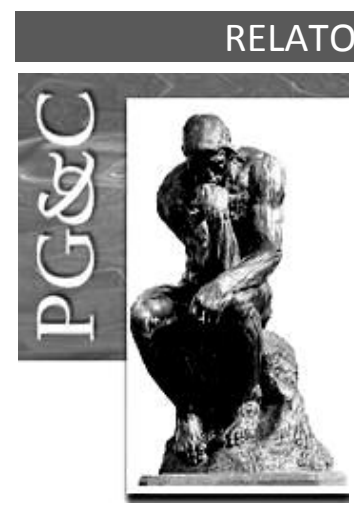

\title{
MATURIDADE DE GESTÃO DO CONHECIMENTO: UMA REVISÃO SISTEMÁTICA DA LITERATURA PARA APOIAR O DESENVOLVIMENTO DE NOVOS MODELOS DE AVALIAÇÃO
}

\author{
Rodrigo Kraemer \\ Mestrando em Engenharia e Gestão do Conhecimento pela Universidade \\ Federal de Santa Catarina, Brasil. \\ E-mail: rodrigokop@gmail.com \\ Patrícia de Sá Freire \\ Doutora em Engenharia e Gestão do Conhecimento pela Universidade \\ Federal de Santa Catarina. Professora da Universidade Federal de Santa \\ Catarina, Brasil. \\ E-mail: patricia.sa.freire@ufsc.br
}

\section{João Artur de Souza}

Doutor em Engenharia de Produção pela Universidade Federal de Santa Catarina, Brasil. Professor da Universidade Federal de Santa Catarina, Brasil.

E-mail: jartur@egc.ufsc.br

\section{Gertrudes A. Dandolini}

Doutora em Engenharia de Produção pela Universidade Federal de Santa Catarina, Brasil. Professora da Universidade Federal de Santa Catarina, Brasil.

E-mail: gertrudes.dandolini@ufsc.br

\begin{abstract}
Resumo
Muitos modelos de maturidade de gestão do conhecimento foram criados no ambiente acadêmico e por consultorias e a maioria deles é baseada no Capability Maturity Model. A diversidade e escala de publicações fez com que o campo de pesquisa fique cada vez mais confuso, sendo necessário analisar e estruturar o campo de uma forma sistemática. $O$ objetivo do artigo é analisar as publicações científicas da última década sobre maturidade de gestão do conhecimento. Para tal, foi realizada uma pesquisa exploratória descritiva com base em uma revisão sistemática da literatura, consultando as bases internacionais Scopus e Web of Science. As publicações analisadas puderam ser categorizadas entre cinco diferentes abordagens sobre maturidade de gestão do conhecimento: (1) que propõe um novo modelo; (2) que relacionam maturidade com outra variável; (3) que analisam a mudança dos níveis de maturidade; (4) que aplicam em diversas organizações; (5) que analisam os modelos previamente existentes. A análise desse campo mostra que o tema maturidade de GC é um campo fértil de pesquisa que oferece várias possibilidades. Muitas críticas aos modelos se referem à excessiva carga depositada na tecnologia, mas novos caminhos com foco em pessoas e aprendizagem estão surgindo. Porém, ainda se faz necessário mais estudos teórico-empíricos para verificação de consistência e validação desses modelos. Outra contribuição é a identificação da lacuna de conhecimentos sobre a comparação da maturidade de gestão do conhecimento entre empresas e países, visto que os modelos poderiam ser utilizados para tal.
\end{abstract}

Palavras-chave: Maturidade. Modelo de maturidade. Gestão do conhecimento. Revisão sistemática da literatura.

Perspectivas em Gestão \& Conhecimento, João Pessoa, v. 7, Número Especial, p. 66-79, mar. 2017. http://periodicos.ufpb.br/ojs2/index.php/pgc. ISSN: 2236-417X. Publicação sob Licença (cc) EY-NC-ND 


\title{
KNOWLEDGE MANAGEMENT MATURITY: A SYSTEMATIC REVIEW OF THE LITERATURE TO SUPPORT THE DEVELOPMENT OF NEW EVALUATION MODELS
}

\begin{abstract}
Many practitioners and researchers have developed Knowledge Management maturity models that were created in academic and consulting and most of them are based on the Capability Maturity Model. The diversity and range of publications make the search field becomes increasingly confused, being necessary to analyze and structure the field in a systematic way. The purpose of this article is to analyze the scientific publications of the last decade about knowledge management maturity. For such a descriptive exploratory study based on a systematic review of the literature was performed by consulting international databases Scopus and Web of Science. The analyzed publications could be categorized in five different approaches to knowledge management maturity, that listed: (1) proposing a new model; (2) relating maturity with another variable; (3) analyze the changing levels of maturity; (4) apply in various organizations; (5) analyzing the previously existing designs. The analysis of this field shows that the knowledge management maturity is a fertile field of research that offers several possibilities. Criticisms identified on existing models refer to the excessive focus on technology, but new ways to focus on people are emerging. However, still needed more theoretical and empirical studies for consistency check and validation of these models. Another contribution is to identify the knowledge gap on the comparison of the maturity of knowledge management between companies and countries, since the models could be used for such.
\end{abstract}

Keywords: Maturity. Maturity model. Kknowledge management. Systematic review of literature.

\section{INTRODUÇÃO}

Mesmo sem saber bem ao certo o que é a Gestão do Conhecimento - GC, muitas organizações utilizam ferramentas relacionadas, por isso uma das primeiras ações é avaliar a GC para uma implantação eficaz (FREIRE et al., 2013). Uma forma de avaliação é com a utilização de modelos de maturidade.

Muito modelos de maturidade de GC foram criados no ambiente acadêmico e por consultorias (KURIAKOSE et al., 2010) e a maioria deles á baseada no Capability Maturity Model - CMM (OLIVA, 2014). Alguns autores procuram integrar os principais modelos de maturidade de GC, mas como vários modelos têm sido desenvolvidos por empresas de consultoria (KURIAKOSE et al., 2010) e há poucos relatos da aplicação deles (OLIVEIRA et al., 2014), possuem muitas lacunas teóricas que dificultam o desenvolvimento da pesquisa acadêmica (ARIAS-PÉREZ; DURANGO-YEPES, 2015).

A quantidade de publicações relacionadas com a maturidade é crescente, principalmente com foco em engenharia de software. Entretanto, segundo Wender (2012), a diversidade de publicações propondo diferentes escalas faz com que o campo de pesquisa em modelo de maturidade fique cada vez mais confuso, sendo necessário analisar e estruturar o campo de uma forma mais sistemática. Assim, torna-se importante promover estudos que se proponham a analisar e comparar os trabalhos acadêmicos sobre o assunto.

Neste contexto, esse trabalho visa responder a seguinte questão de pesquisa: como as pesquisas acadêmicas têm trabalhado com o tema maturidade de GC na última década? Para responder a esta questão elaborou-se como objetivo: analisar as características bibliométricas da última década sobre maturidade de GC para identificar as tendências e as lacunas temáticas das publicações indexadas em base de dados internacional.

O artigo está estruturado da seguinte forma, a próxima seção é apresentada uma discussão teórica, a seção 3 descreve a metodologia utilizada, a seção 4 mostra os resultados encontrados que são discutidos na seção 5 e finalizado com as considerações finais e sugestões de estudo futuros.

Perspectivas em Gestão \& Conhecimento, João Pessoa, v. 7, Número Especial, p. 66-79, mar. 2017. 


\section{MODELOS DE MATURIDADE}

Para compreender as diferentes abordagens das publicações levantadas e ser possível a sua categorização, fez-se necessário uma compreensão rápida sobre a origem dos modelos de maturidade. A seguir, em poucos parágrafos, antes de apresentar os resultados da revisão sistemática, descreve-se o resumo sobre essa história.

Os modelos de maturidade surgiram com a engenharia de software, como um modelo que representa os estágios de desenvolvimento de uma empresa para implementação de processos (DALKIR, 2013). No geral, a literatura apresenta vários modelos de maturidade de GC, sendo a maioria baseada no CMM (OLIVA, 2014). O modelo CMM foi criado pelo Software Engineering Institute - SEl, e o processo de desenvolvimento de software foi dividido em cinco níveis de maturidade com características distintivas, fornecendo referências para os gestores e mostrando os passos de melhoria contínua do processo (FENG, 2006). O Capability Maturity Model Integration - CMMI é um framework derivado do CMM que contém as melhores práticas para o desenvolvimento de produtos e serviços e combina conceitos de desenvolvimento de software, engenharia de sistemas e desenvolvimento de produtos (WENDLER, 2012).

Com base nas características de modelos de maturidade, diversos modelos foram criados para diversas áreas, como gestão financeira, gestão de caixa, gestão de projetos, gestão da inovação, gestão da qualidade, design de produto, gestão de serviços, gestão de relacionamento com o cliente e gestão de processos de negócios (XIAO et al. 2012).

Os modelos de maturidade possuem as seguintes propriedades (KLIMKO, 2001): a evolução de uma entidade é descrita com base em vários níveis de maturidade; cada nível de maturidade é caracterizado em atributos únicos; níveis são apresentados sequencialmente; e a entidade avança consecutivamente de um nível para outro, sem omitir qualquer nível, porque cada estágio inferior cria uma necessidade para o próximo nível de desenvolvimento.

Quanto aos modelos de maturidade de GC, estes são utilizados para superar a natureza estática das avaliações de GC e podem formalmente capturar o processo de desenvolvimento avaliando em que medida a GC é explicitamente definida, gerenciada e controlada (CHEN; FONG, 2012).

A utilização de modelos de maturidade para a GC é importante por quatro razões, conforme descrito por Serenko et al. (2014). Em primeiro lugar, a implementação bemsucedida de iniciativas de GC exige uma abordagem holística, sistemática e estruturada para desenvolver, medir e melhorar continuamente os processos organizacionais relacionados. Em segundo lugar, modelos de maturidade servem como uma ferramenta eficaz para facilitar a governança de GC em toda a organização. Em terceiro lugar, auxilia a identificar barreiras à implementação da GC. Em quarto lugar, modelos de maturidade podem facilitar o planejamento de curto e longo prazo.

Enfim, em geral, os modelos de maturidade descrevem uma evolução de iniciativas de GC organizacionais ao longo do tempo (SERENKO ET AL, 2014). O que será possível compreender pela revisão sistemática da literatura realizada por esta pesquisa.

\section{METODOLOGIA}

Este artigo se baseou em uma revisão sistemática da literatura sobre maturidade em GC. O método de revisão sistemática de literatura pode ser explicado como uma síntese de estudos primários com objetivos e métodos bem explicitados, realizados através de uma metodologia clara e reprodutível (GREENHALGH, 1997).

Perspectivas em Gestão \& Conhecimento, João Pessoa, v. 7, Número Especial, p. 66-79, mar. 2017. 
A busca sistemática ocorreu na Web of Science - WoS e Scopus. Na WoS foi selecionada a Social Sciences Citation Index (SSCl) e na Scopus a Social Science \& Humanities. Em ambas as bases foi pesquisado no período de 2005 até a data de 01/06/2016.

Foram utilizados os termos relacionados ao tema, como maturity e maturity model além de knowledge management e também sua abreviatura $\mathrm{km}$, conforme mostrado na Tabela 1. Os artigos foram agrupados por base e importados para o software EndNote ${ }^{\circledR}$, onde foram novamente agrupados e retirados os duplicados, totalizando 66 artigos.

Tabela 1 - Termos pesquisados por base

\begin{tabular}{l|c|c}
\hline Palavras-chave & WoS & Scopus \\
\hline "knowledge management maturity" & 8 & 45 \\
\hline "km maturity" & 8 & 23 \\
\hline "maturity model for knowledge management" & 0 & 1 \\
\hline "maturity model for km" & 1 & 6 \\
\hline "maturity model of knowledge management" & 1 & 4 \\
\hline "maturity model of km" & 0 & 0 \\
\hline Total & 12 & 63 \\
\hline Total sem duplicados & & 66 \\
\hline
\end{tabular}

Fonte: Autoria própria

Foram lidos os resumos dos artigos para verificar a relação com o tema e foram eliminados 30 (trinta) artigos que não tratavam de maturidade de GC, que apenas tangenciavam sobre o tema ou que tratavam de maturidade em processos específicos ou ferramentas de GC, restando 36 (trinta e seis) artigos para leitura completa.

Os artigos foram tabulados em uma planilha para identificar as semelhanças, foram lidos e categorizados com base nas similaridades encontradas nos objetivos dos artigos. Foram ainda incorporados dois artigos (KRUGER; SNYMAN, 2007; ARIAS-PÉREZ; DURANGO-YEPES, 2015) encontrados pelas referências pertencentes ao estudo, restando 38 (trinta e oito) artigos. Desta forma, esses 38 (trinta e oito) artigos se configuraram na amostra deste trabalho.

\section{ANÁLISE DOS RESULTADOS DA BUSCA SISTEMÁTICA}

As publicações aconteceram em todos os anos pesquisados com algumas oscilações, mantendo uma média de três publicações por ano. O Gráfico 1 mostra que ocorreu um pico em 2012 com nove artigos, e os anos com mais publicações foram 2015 com cinco artigos além de 2011 e 2014 com quatro artigos. Em 2016, até final de maio havia uma publicação.

Perspectivas em Gestão \& Conhecimento, João Pessoa, v. 7, Número Especial, p. 66-79, mar. 2017. 
Gráfico 1 - Ano de publicação

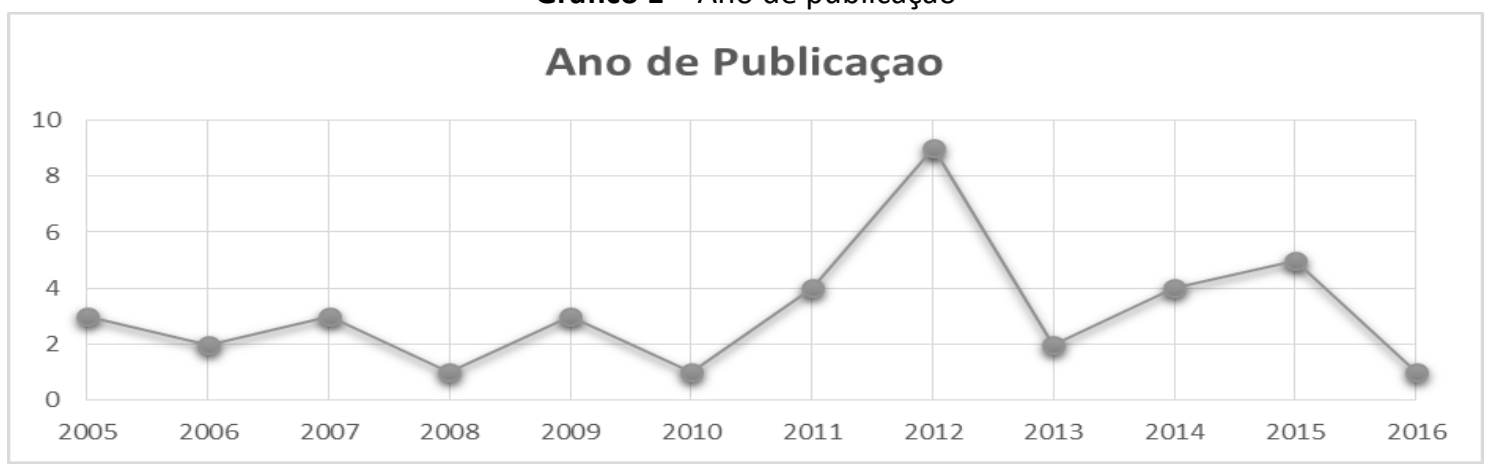

Fonte: Dados da pesquisa, 2016

Do total de 38 (trinta e oito) publicações, 20 (vinte) são de artigos publicados em journals e 18 (dezoito) artigos de conferências. A principal conferência foi a ECKM - European Conference on Knowledge Management que trouxe oito artigos. Os Journals que mais publicaram artigos são o Expert Systems with Applications, o Journal of Information and Knowledge Management e o Journal of Knowledge Management.

A seleção foi composta por 78 (setenta e oito) autores, sendo que alguns se destacaram com maior número de publicações, como Bernard, P., Ekionea, J. P. B., Kruger, C. J. e Plaisent, M. com quatro artigos cada um e Johnson, R. D., e Sujanova, J. com três artigos cada. Outros dez autores tiveram dois artigos na seleção e os demais 62 (sessenta e dois) autores apenas um artigo.

Os artigos mais citados na base Scopus foram Salojärvi et al. (2005) com 98 (noventa e oito) citações, Robinson et al. (2005) com 63 (sessenta e três) citações e Robinson et al. (2006) com 48 (quarenta e oito) citações. Na base Web of Science os mais citados foram Hsieh et al. (2009) com 27 (vinte e sete) citações, Lin et al. (2012) com dez citações e Mehta et al. (2007) com nove citações.

Com base nos objetivos dos artigos foram construídas cinco categorias, conforme mostra o Quadro 1.

Quadro 1 - Categorias dos artigos

\begin{tabular}{|c|c|}
\hline CATEGORIA & ARTIGOS \\
\hline Propõe um novo modelo & $\begin{array}{l}\text { (HUNG et al., 2005); (ROBINSON et al., 2005); (ROBINSON et al., 2006); } \\
\text { (FENG, 2006); (EKIONEA et al., 2007); (EKIONEA et al., 2011A); (EKIONEA } \\
\text { et al., 2011B); (EKIONEA et al., 2012); (KRUGER; SNYMAN, 2007); } \\
\text { (GRUNDSTEIN, 2008); (HSIEH et al., 2009); (PEE; KANKANHALLI, 2009); } \\
\text { (FENGJU; XIAOJING, 2011); (SERNA, 2012); (CHEN; FONG, 2012); (YANG; } \\
\text { YU, 2013); (OLIVEIRA; PEDRON, 2014); (ARIAS-PÉREZ; DURANGO-YEPES, } \\
\text { 2015); (GALATI, 2015) }\end{array}$ \\
\hline $\begin{array}{l}\text { Relaciona a maturidade } \\
\text { com outra variável }\end{array}$ & $\begin{array}{c}\text { (KRUGER; JOHNSON, 2011); (HARTONO et al., 2016); (SALOJÄRVI et al., } \\
\text { 2005); (CHAN ET AL, 2012); (PÉREZ, 2013); (HARLOW, 2014) }\end{array}$ \\
\hline $\begin{array}{l}\text { Analisa as mudanças dos } \\
\text { níveis de maturidade }\end{array}$ & $\begin{array}{l}\text { (MEHTA et al., 2007); (KRUGER; JOHNSON, 2009); (LIN et al., 2012); } \\
\text { (XIAO et al., 2012); (FOLLADOR; TRABASSO, 2015) }\end{array}$ \\
\hline $\begin{array}{l}\text { Aplica em diversas } \\
\text { empresas }\end{array}$ & $\begin{array}{l}\text { (KRUGER; JOHNSON, 2010); (GABRIS et al., 2012); (SUJANOVA et al., } \\
\text { 2012); (SUJANOVA et al., 2015); (OLIVEIRA et al., 2014); (LEE; WONG, } \\
\text { 2015); (SERENKO et al., 2014) }\end{array}$ \\
\hline $\begin{array}{l}\text { Analisa os modelos } \\
\text { existentes }\end{array}$ & (ESCRIVÃO; SILVA, 2012) \\
\hline
\end{tabular}

Fonte: Dados da pesquisa, 2016

Perspectivas em Gestão \& Conhecimento, João Pessoa, v. 7, Número Especial, p. 66-79, mar. 2017. 
A primeira categoria é composta de artigos que propõem um novo modelo teórico e aplicam em uma ou mais organizações ou constroem um modelo baseado nos anteriores com algumas modificações. Foram encontrados 15 (quinze) diferentes modelos propostos na última década, sendo 11 (onze) baseados no CMM (ROBINSON et al., 2005; HUNG et al., 2005; FENG, 2006; EKIONEA et al., 2007; KRUGER; SNYMAN, 2007; HSIEH et al., 2009; PEE; KANKANHALLI, 2009; FENGJU; XIAOJING, 2011; YANG; YU, 2013; OLIVEIRA; PEDRON, 2014; ARIAS-PÉREZ; DURANGO-YEPES, 2015), um baseado no BSC (CHEN; FONG, 2012), um baseado no COBIT (GRUNDSTEIN, 2008) e outros dois artigos que não possuem um modelo de base definido (GALATI, 2015; SERNA, 2012).

$\mathrm{Na}$ segunda categoria encontram-se trabalhos que relacionam a maturidade em GC com outra variável, como "crescimento" (SALOJÄRVI et al., 2005), "capital intelectual" (CHAN et al., 2012), "inovação" (PÉREZ, 2013; HARLOW, 2014) e "desempenho organizacional" (KRUGER; JOHNSON, 2011; HARTONO et al., 2016).

$\mathrm{Na}$ categoria que analisam as mudanças nos níveis de maturidade, são encontrados artigos que descrevem as dificuldades encontradas em relação à análise custo-benefício de se chegar ao último nível de maturidade (MEHTA ET AL, 2007), analisa as mudanças de níveis em diferentes agrupamentos empresariais para perceber as variações no crescimento dos setores (KRUGER; JOHNSON, 2009), identifica barreiras para cada nível (LIN et al., 2012), analisa a implementação de áreas-chave para a mudança de nível (XIAO ET AL, 2012) ou mostra que uma nova estrutura de pesquisa pode não alterar a maturidade (FOLLADOR; TRABASSO, 2015).

Os estudos que analisam a aplicabilidade em diversas empresas procuram encontrar entendimentos comuns (GABRIS et al., 2012; SUJANOVA et al., 2012; SUJANOVA et al., 2015), tendências (KRUGER; JOHNSON, 2010) ou entender como a GC está presente nas empresas ou setores de um país (OLIVEIRA et al., 2014; LEE; WONG, 2015; SERENKO et al., 2014).

A categoria que analisa os modelos de maturidade é composta por apenas um artigo que tece críticas ainda não resolvidas na criação de modelos de maturidade (ESCRIVÃO; SILVA, 2012).

\section{DISCUSSÃO}

Após ter categorizado as publicações levantadas, nessa seção são analisadas em mais profundidade cada uma das categorias, tentando promover um diálogo subjetivo entre as propostas dos autores. Analisa-se como a maturidade se relaciona com outras variáveis; como são analisadas as mudanças de níveis; como foram realizadas as aplicações em diversas empresas; para então verificar as críticas encontradas aos modelos atuais e mostrar alguns modelos selecionados.

\subsection{A maturidade relacionada com algumas variáveis}

Alguns estudos utilizam a maturidade em GC correlacionando-a com outras variáveis, encontrando ou não correlação. A relação entre maturidade e desempenho organizacional foi realizada em empresas de grande porte na África do Sul com alguma correlação encontrada (KRUGER; JOHNSON, 2011) e em indústrias de construção na indonésia com relação positiva e significativa (HARTONO et al., 2016). A relação entre maturidade e crescimento foi estudada em pequenas e médias empresas - PMEs eslovacas encontrando correlação positiva (SALOJÄRVI et al., 2005). Estudando empresas chinesas, Chan et al (2012) não encontrou correlação significativa entre maturidade e capital intelectual. Estudando maturidade com o desempenho inovador, Pérez (2013) não encontrou relação significativa na inovação de produto, mas encontrou nas inovações mais intangíveis como processo organizacional e

Perspectivas em Gestão \& Conhecimento, João Pessoa, v. 7, Número Especial, p. 66-79, mar. 2017. 
marketing. Já o estudo de Harlow (2014) não encontrou relação entre maturidade e inovação em empresas americanas.

\subsection{Mudança de níveis}

As mudanças nos níveis de maturidade foram estudadas em relação ao custo-benefício na mudança de nível, analisando a diferença de mudança de nível entre diferentes setores, encontrando barreiras diferentes para cada nível de maturidade ou facilitadores para a mudança, além do impacto da implantação de uma nova estrutura de pesquisa e desenvolvimento na mudança do nível de maturidade.

Mehta et al. (2007) descreveu o programa de GC da Infosys Technologies e o questionamento do Conselho de Administração sobre o investimento financeiro da empresa para passar ao último nível de maturidade em GC. Esse questionamento levou o CEO a repensar nas métricas utilizadas para avaliar as melhorias nos fluxos de conhecimento organizacional tornando-as mais tangíveis.

Em relação às diferenças de mudança entre setores empresariais, Kruger e Johnson (2009) mostraram as diferenças no crescimento da maturidade de GC em diferentes setores empresariais na África do Sul, encontrando crescimento elevado em: construção, materiais de construção e empresas de mineração; crescimento moderado em: bens de consumo e serviços públicos, bancos e seguros, automotivo e transportes, governo e empresas de TIC; e finalmente baixo crescimento em instituições de ensino, todas em um período de 5 anos.

Realizando um estudo longitudinal em 7 empresas, Lin et al. (2012) perceberam que as barreiras ao fluxo de conhecimento eram diferentes nos níveis de maturidade. Os autores utilizaram 37 barreiras encontradas na literatura e as classificaram em quatro espécies: existência (as barreiras nunca mudam quando a GC desenvolve), queda (barreiras diminuem), aumento (barreiras crescem), e aleatória (barreiras dinâmicas).

Xiao et al. (2012) realizam uma análise de diversos modelos de maturidade em GC e das diferentes áreas-chave de processo listadas em cada modelo e identificam 4 mais utilizadas - pessoas, tecnologia, cultura e processo. Analisando os mecanismos de transição de nível de maturidade, concluem que a implementação das áreas-chave de processos é a base das transições de níveis de maturidade.

Em alguns casos, as organizações procuram criar áreas na estrutura para desenvolver seu potencial de gestão do conhecimento, mas nem sempre isso auxilia. Follador e Trabasso (2015) mostraram que a maturidade em GC não foi alterada com a implantação de uma estrutura de gestão de ciência e tecnologia em um departamento de testes de aviação no Brasil.

\subsection{Aplicação em diversas empresas}

A maturidade também pode ser utilizada aplicando em diversas empresas de um país para encontrar entendimentos comuns, tendências, entender como a GC está presente nos setores ou empresas de um país ou simplesmente para testar novos modelos.

Comparando diversas empresas, Gabris et al. (2012) identificaram desproporções entre o entendimento sobre a GC e a implementação de GC, e Sujanova et al. (2012) encontraram disparidades entre a estratégia e a implementação de GC, ambos em grandes empresas eslovacas. Repetindo a pesquisa em 2015, Sujanova et al. (2015) encontraram avanços, percebendo que as empresas começaram a tomar consciência da importância dos ativos de conhecimento, concentrando-se em processos de conhecimento intensivo e inovação.

Perspectivas em Gestão \& Conhecimento, João Pessoa, v. 7, Número Especial, p. 66-79, mar. 2017. 
Kruger e Johnson (2010) perceberam uma tendência para o estabelecimento de princípios da GC em todos os setores industriais sul-africanos e encontraram elementos que facilitam ou influenciam a capacidade de GC nas empresas.

Com relação às PMEs, Oliveira et al. (2014) encontraram baixos níveis de maturidade em TI, conhecimento explícito, parceiros, criação e armazenamento de conhecimento e nível alto em apoio da alta gestão em empresas brasileiras. Já Lee e Wong (2015) afirmaram que empresas menores dependem mais de conhecimento externo em comparação com as médias empresas, e nas microempresas a principal fonte de conhecimento é de seus proprietáriosgerentes. Aplicando em cooperativas de crédito norte-americanas, Serenko et al. (2014) encontraram que mesmo sendo organizações intensivas em conhecimento, nenhuma possuía uma estratégia formal de GC, apenas iniciativas isoladas em algum departamento.

A aplicação em organizações pode também foi realizada para testar novos modelos. Os modelos propostos na última década foram aplicados em bancos (HUNG et al., 2005; FENG, 2006), em grandes empresas de construção do Reino Unido (ROBINSON ET AL, 2006) ou de Hong Kong (CHEN; FONG, 2012) e em organizações públicas (PEE; KANKANHALLI, 2009; EKIONEA et al., 2012). Em alguns casos os setores não são específicos, sendo empregadas em diversas empresas, em Taiwan (HSIEH et al., 2009) ou grandes empresas colombianas (ARIASPÉREZ; DURANGO-YEPES, 2015). Também há modelos aplicados em PMEs brasileiras (OLIVEIRA et al., 2014).

\subsection{Críticas aos modelos de maturidade}

Muitos modelos têm sido criticados porque as suas ferramentas de avaliação são proprietárias ou não especificadas, tornando a sua avaliação empírica difícil (PEE; KANKANHALLI, 2009). Outros foram criticados por integrarem apenas facilitadores, sendo necessário também se basear nos processos de GC (FENG, 2006).

Muitos modelos têm uma perspectiva linear, sequencial, determinista e invariável do desenvolvimento da GC, e têm sido criticados porque apesar de apresentarem boas características para definir um processo de desenvolvimento de produto, eles não são bons para a definição do desenvolvimento da organização (ESCRIVÃO; SILVA, 2012). Os modelos propostos mostram a dificuldade em provar uma única sequência de etapas e não há um consenso sobre o número de estágios (ESCRIVÃO; SILVA, 2012). Além disso, quase todos os modelos condicionam a maturidade com o último nível (ESCRIVÃO; SILVA, 2012), quando, na verdade, há casos em que os custos não compensam os benefícios de manter-se na última etapa, como por exemplo o caso da Infosys apresentado por Mehta et al. (2007).

Sistemas de TIC são muitas vezes vistos como sendo sistemas de GC (KRUGER; JOHNSON, 2009). No entanto, a GC possui algumas especificidades, é menos estruturada que os processos de software, suas atividades são menos padronizadas e os resultados menos quantificáveis (PEE; KANKANHALLI, 2009).

Uma alta dependência de tecnologia resultou na maturidade de GC sendo derivada do modelo CMM (KRUGER; JOHNSON, 2009). Esses modelos colocam muita ênfase na tecnologia, negligenciando importantes dimensões estratégicas (KRUGER; SNYMAN, 2007) e desconsiderando processos de aprendizagem (ESCRIVÃO; SILVA, 2012).

No entanto, não apenas os modelos baseados no CMM recebem essas críticas. Realizando uma comparação de vários modelos existentes, Pee e Kankanhalli (2009) classificaram em dois grupos, dependendo se eram ou não desenvolvidos com base no CMM e observaram várias características comuns entre os dois grupos de modelos, como a descrição dos níveis e a definição das área-chaves. Ou seja, mesmo não se baseando conscientemente, os modelos criados possuíam características comuns com os baseados no CMM.

Perspectivas em Gestão \& Conhecimento, João Pessoa, v. 7, Número Especial, p. 66-79, mar. 2017. 


\subsection{Novos modelos da última década}

Conforme já mencionado, foram encontrados 15 (quinze) modelos que foram propostos na última década. Assim, foram selecionados cinco modelos que são explanados, sendo um baseado no COBIT, três no CMM, dois não baseados em outros modelos de medição e um criado procurando misturar modelos diferentes.

Conforme já apontado por Oliva (2014), a maioria dos novos modelos apresentados foi baseada no CMM. Muitos autores, ao criarem novos modelos de maturidade, criticam o foco na tecnologia, mas continuam utilizando como referência modelos criados para esse fim. Por exemplo Grundstein (2008) afirma que seu modelo é definido como sociotécnico e baseado mais em pessoas que em tecnologia, mas o desenvolve baseado no COBIT que é um modelo de governança de TI. Isso mostra que por mais que o autor queira adicionar pessoas ou cultura ao seu modelo, sua base de referência é fortemente marcada por meios técnicos e não por meios sociais.

Baseado no CMM mas também em gestão da qualidade (Quality Management Maturity Grid), Hsieh et al. (2009) propõem o Knowledge Navigator Model (KNM). Criticando a falta de um modelo com itens e procedimentos mais detalhados, o modelo apresenta três dimensões de análise: cultura, TI e processos de GC. O questionário é composto por 68 atividades de GC divididos em 16 áreas-chave. Os autores aplicaram o modelo em 30 empresas de Taiwan.

Afirmando que é necessário criar modelos mais holísticos incluindo outras perspectivas, dimensões ou áreas-chave, Pee e Kankanhalli (2009) apresentam o General KM Maturity Model (G-KMMM). Cada nível de maturidade é caracterizado por três áreas-chave: pessoas, processos e tecnologia. O modelo é flexível e pode ser utilizado em unidades, departamentos ou organizações, podendo medir as diferenças entre as unidades organizacionais, porém os autores ressaltam que o foco é no uso em unidades intensivas em conhecimento. $\mathrm{O}$ modelo foi aplicado em uma organização de sistema de informação de uma universidade pública. Os autores disponibilizam a ferramenta de avaliação para que outros pesquisadores possam utilizar e validar, pois criticam que muitos modelos não são validados porque são proprietários ou não especificados.

Tendo como objetivo propor um modelo de maturidade que relacione a GC com a capacidade absortiva, inovação e desempenho organizacional, Oliveira e Pedron (2014) propõem um modelo com quatro fatores principais (com 10 constructos): o ambiente interno (apoio da alta administração, tecnologia da informação), o conteúdo (conhecimento tácito e explícito), os processos (criação, armazenamento e compartilhamento), e o ambiente externo (clientes, parceiros e fornecedores). Os autores também criam uma hierarquia dos fatores. 0 modelo é aplicado posteriormente em PMEs em Oliveira et al. (2014).

Discutindo que a GC deve ser centrada na interação humana ao invés da informação e pensada enquanto processo e não como resultado, Serna (2012) afirma que o problema não é apenas a predominância da tecnologia, mas que os problemas apresentados pelos modelos de maturidade são provocados pela visão de mundo dos autores. Criticando os modelos que seguem a visão funcionalista, de acordo com a classificação de Morgan (1980), Serna (2012) propõe um modelo teórico baseado em uma perspectiva interpretativista. Afirmando que esta visão está se tornando dominante na GC, ele se baseia nas premissas que a realidade é um fenômeno subjetivo, que o conhecimento é dependente do contexto, que a aprendizagem é uma prática social baseado nas pessoas e que o conhecimento não pode ser armazenado pois depende de cada situação específica. O modelo é construído fazendo um paralelo com os objetivos de aprendizagem da taxonomia de Bloom, que propôs três domínios de objetivos educacionais: cognitivo (conhecimento), afetivo (atitude) e psicomotor (habilidade). O autor então agrupa o domínio cognitivo em 4 dimensões: gestão de recursos, (Resources

Perspectivas em Gestão \& Conhecimento, João Pessoa, v. 7, Número Especial, p. 66-79, mar. 2017. 
management), gestão analítica (Analytic management), gestão de significado (Significant management) e gestão da ação (Active management). Os níveis são apresentados em um quadro referencial, mas o modelo não é aplicado.

Tendo como objetivo desenvolver um framework teórico combinando diferentes perspectivas de GC e da aprendizagem organizacional, Galati (2015) construiu um modelo baseado em três dimensões - fontes de conhecimento, processos de implementação de GC e consciência da estratégia de GC. Na primeira dimensão a fonte pode ser interna ou externa e, neste caso, relacionada à capacidade absortiva. Na segunda dimensão, o processo de implementação de GC é definido como um processo de mudança organizacional, através das fases: descongelando, quando se implanta um senso de urgência em mudanças; movendo-se, que implica na implementação da mudança em si; e institucionalizando, que se refere à consolidação dos avanços obtidos para não permitir o retorno à situação anterior. Já a dimensão de consciência de GC possui 4 níveis: sem estratégia, quando a organização não controla os fluxos de conhecimento; estratégia de codificação quando a organização apenas explicita o conhecimento: estratégia de simbiose, onde a organização gerencia tanto o explícito quanto o tácito; e organização de aprendizagem, quando a aprendizagem organizacional é o foco principal de toda organização.

Arias-Pérez e Durango-Yepes (2015) constroem um modelo de maturidade a partir das perspectivas funcionalista e interpretativa, utilizando como base os modelos desenvolvidos por Pee e Kankanhalli (2009) e Serna (2012). O modelo é composto por cinco níveis de maturidade e quatro áreas-chave (com variáveis em cada área): pessoas e organização (trust, incentive systems, structure, strategy); processos (creation, collection, exchange, application); tecnologia (technological infrastructure integration, $\mathrm{km}$ applications, attitude towards ICT); e interpretação (meaning management, action management). Cada variável citada pelos autores possui níveis de maturidade próprios. O modelo foi aplicado em grandes empresas na Colômbia.

\section{CONSIDERAÇÕES FINAIS}

A presente pesquisa vem contribuir com a constatação que as pesquisas acadêmicas têm trabalhado com o tema maturidade de GC na última década através de cinco abordagens diferentes: criando um novo modelo, relacionando a maturidade com outra variável, analisando a mudança nos níveis de maturidade, aplicando em diversas empresas ou simplesmente analisando os modelos propostos.

A maioria dos trabalhos procura desenvolver um novo modelo, e a maioria dos modelos são ainda baseados no CMM. Ainda há pouca referência à conceitos relacionados à semiótica e teorias de aprendizagem, que são fundamentais para uma visão mais completa da GC (PÉREZ, 2013), mas uma tendência ao desenvolvimento de modelos diferenciados, seguindo lógicas diferentes pode ser observada. Modelos baseados na aprendizagem individual (SERNA, 2012) e na aprendizagem organizacional (GALATI, 2015) tem surgido, além de um modelo misto que procura conjugar as visões funcionalista e interpretativista (ARIASPÉREZ; DURANGO-YEPES, 2015).

A análise desse campo mostra que o tema maturidade de GC é um campo fértil de pesquisa que oferece várias possibilidades. Muitas críticas aos novos modelos se referem à excessiva carga depositada na tecnologia, mas novos caminhos com foco em pessoas estão surgindo, precisando de mais estudos para testar esses modelos. A maturidade pode ser utilizada para avaliar diversas empresas ou setores de um país, mas ainda não existem comparações internacionais. E a investigação acerca das barreiras e facilitadores para as mudanças de níveis de maturidade ainda é um tema emergente.

Perspectivas em Gestão \& Conhecimento, João Pessoa, v. 7, Número Especial, p. 66-79, mar. 2017. 
Como conclusão final, pode-se destacar a importância do estudo realizado para apoiar o desenvolvimento de novos modelos de avaliação de maturidade em gestão do conhecimento.

\section{REFERÊNCIAS}

ARIAS-PÉREZ, J. E.; DURANGO-YEPES, C. M. Exploring knowledge management maturity from funcionalist and interpretivist perspectives. Entramado, v. 11, p. 94-104, 2015.

CHAN, K. H.; CHU, S. K. W.; WU, W. W. Y. Exploring the correlation between knowledge management maturity and intellectual capital efficiency in mainland chinese listed companies. Journal of Information and Knowledge Management, v. 11, n. 3, 2012.

CHEN, L.; FONG, P. S. W. Revealing performance heterogeneity through knowledge management maturity evaluation: A capability-based approach. Expert Systems with Applications, v. 39, n. 18, p. 13523-13539, Dec 2012.

DALKIR, K. Knowledge management in theory and practice. Taylor and Francis, 2013. 1-356.

EKIONEA, J. P. B.; BERNARD, P.; PLAISENT, M. Towards a maturity model of knowledge management competences as an organisational capability. 2nd International Conference on EBusiness and E-Government, ICEE 2011, 2011, Shanghai. p.9267-9271.

EKIONEA, J. P. B.; FILLION, G.; PLAISENT, M.; BERNARD, P. Towards an integrated maturity model of knowledge management capabilities. 2nd International Conference on E-Business and E-Government, ICEE 2011, 2011, Shanghai. p.9272-9275.

. Using the soft system methodology for designing an integrated and inter-firm knowledge management capabilities maturity model. 13th European Conference on Knowledge Management, ECKM 2012, 2012, Cartagena. p.108-117.

EKIONEA, J. P. B.; PLAISENT, M.; BERNARD, P. Developing knowledge management competences as an organizational capability for business performance. 8th European Conference on Knowledge Management, ECKM 2007, p. 131-138, 2007.

ESCRIVÃO, G.; SILVA, S. L. Knowledge management maturity: Critical reflections and identification of gaps. 13th European Conference on Knowledge Management, ECKM 2012, 2012, Cartagena. p.1542-1552.

FENG, J. A knowledge management maturity model and application. PICMET '06 TECHNOLOGY MANAGEMENT FOR THE GLOBAL FUTURE, 2006, Istanbul. p.1251-1255.

FENGJU, X.; XIAOJING, D. Research on the innovative enterprise knowledge management based on maturity model. $2011 \mathrm{In}$ : INTERNATIONAL CONFERENCE ON PRODUCT INNOVATION MANAGEMENT, ICPIM 2011, 2011, Wuhan. p.741-744.

FOLLADOR, R. C.; TRABASSO, L. G. Knowledge Management maturity level in a Brazilian Air Force flight test environment. In: DAIM, T. U. et al. In: PORTLAND INTERNATIONAL CENTER FOR MANAGEMENT OF ENGINEERING AND TECHNOLOGY, PICMET 2015, 2015, Portland State University. p.1296-1304.

Perspectivas em Gestão \& Conhecimento, João Pessoa, v. 7, Número Especial, p. 66-79, mar. 2017. 
GABRIS, P.; BIELIK MARETTOVÁ, M.; PAVLENDA, P.; LICKO, M.; SUJANOVA, J. Knowledge management maturity aspects in industrial enterprises. INTERNATIONAL DAAAM BALTIC CONFERENCE: INDUSTRIAL ENGINEERING, $8^{\text {th }}$. , 2012. DAAAM International. p.454-457.

GALATI, F. At what level is your organization managing knowledge? Measuring Business Excellence, v. 19, n. 2, p. 57-70, 2015.

GREENHALGH, T. Papers that summarise other papers (systematic reviews and meta-analyses). BMJ: British Medical Journal, v. 315, n. 7109, p. 672, 1997.

GRUNDSTEIN, M. Assessing the enterprise's knowledge management maturity level. International Journal of Knowledge and Learning, v. 4, n. 5, p. 415-426, 2008.

HARLOW, H. An empirical comparison study of the effect of chief knowledge management officers and knowledge management systems on innovation and financial outcomes. In: SEQUEIRA et al. In: EUROPEAN CONFERENCE ON KNOWLEDGE MANAGEMENT, ECKM 2014, 2014, Proceedings... Academic Conferences Limited. p.410-418.

HARTONO, B.; RAMADHANI, V. S.; RATNADILLA, Y.; INDARTI, N.; CHAI, K. H. Knowledge management maturity and organizational performance in project-based organizations: Initial evidence from Indonesia. In: INTERNATIONAL CONFERENCE ON INDUSTRIAL ENGINEERING AND ENGINEERING MANAGEMENT, IEEM 2015, 2016, IEEE Computer Society. p.656-660.

HSIEH, P. J.; LIN, B.; LIN, C. The construction and application of knowledge navigator model (KNM (TM)): An evaluation of knowledge management maturity. Expert Systems with Applications, v. 36, n. 2, p. 4087-4100, Mar 2009.

HUNG, Y. H.; CHOU, S. C. T.; CHEN, Y. C. How can we assess knowledge management? Constructing a holistic assessment framework of KM. EUROPEAN CONFERENCE ON KNOWLEDGE MANAGEMENT, $6^{\text {th }}$., ECKM 2005. Proceedings... 2005, p. 264-270, 2005.

KLIMKO, G. Knowledge management and maturity models: building common understanding. EUROPEAN CONFERENCE ON KNOWLEDGE MANAGEMENT, 2., 2001. Proceedings... Bled, Slovenia, 2001. p.269-278.

KRUGER, C. J.; JOHNSON, R. D. Principles in knowledge management maturity: a South African perspective. Journal of Knowledge Management, v. 14, n. 4, p. 540-556, 2010.

. Is there a correlation between knowledge management maturity and organizational performance? VINE, v. 41, n. 3, p. 265-295, 2011.

KRUGER, C. J.; SNYMAN, M. M. M. Guidelines for assessing the knowledge management maturity of organizations. South African Journal of Information Management, v. 9, n. 3, p. 111, 2007.

KURIAKOSE, K.; RAJ, B.; MURTY, S.; SWAMINATHAN, P. Knowledge management maturity models-a morphological analysis. Journal of Knowledge Management Practice, v. 11, n. 3, p. 1-10, 2010.

Perspectivas em Gestão \& Conhecimento, João Pessoa, v. 7, Número Especial, p. 66-79, mar. 2017. 
LIN, C. H.; WU, J. C.; YEN, D. C. Exploring barriers to knowledge flow at different knowledge management maturity stages. Information \& Management, v. 49, n. 1, p. 10-23, Jan 2012.

MEHTA, N.; OSWALD, S.; MEHTA, A. Infosys technologies: Improving organizational knowledge flows. Journal of Information Technology, v. 22, n. 4, p. 456-464, 2007.

MORGAN, G. Paradigms, metaphors, and puzzle solving in organization theory. Administrative science quarterly, p. 605-622, 1980.

OLIVA, F. L. Knowledge management barriers, practices and maturity model. Journal of Knowledge Management, v. 18, n. 6, p. 1053-1074, 2014.

OLIVEIRA, M.; PEDRON, C. D. Maturity model for knowledge management and strategic benefits. In: VIVAS, C. et al, EUROPEAN CONFERENCE ON KNOWLEDGE MANAGEMENT, $15^{\text {th }}$, ECKM 2014, 2014 Proceedings... Academic Conferences Limited. p.748-756.

OLIVEIRA, M.; PEDRON, C. D.; NODARI, F.; RIBEIRO, R. Knowledge management in small and micro enterprises: Applying a maturity model. In: VIVAS, C. et al. In: EUROPEAN CONFERENCE ON KNOWLEDGE MANAGEMENT, 15 ${ }^{\text {th }}$, ECKM 2014, 2014 Proceedings... Academic Conferences Limited. p.757-764.

PEE, L. G.; KANKANHALLI, A. A model of organisational knowledge management maturity based on people, process, and technology. Journal of Information and Knowledge Management, v. 8, n. 2, p. 79-99, 2009.

PÉREZ, J. E. A. Associations between the maturity of knowledge management and innovative performance: Organization and people, and interpretation. Revista Lasallista de Investigacion, v. 9, n. 1, p. 86-95, 2013.

ROBINSON, H. S.; ANUMBA, C. J.; CARRILLO, P. M.; AL-GHASSANI, A. M. STEPS: A knowledge management maturity roadmap for corporate sustainability. Business Process Management Journal, v. 12, n. 6, p. 793-808, 2006.

ROBINSON, H. S.; CARRILLO, P. M.; ANUMBA, C. J.; AL-GHASSANI, A. M. Knowledge management practices in large construction organisations. Engineering, Construction and Architectural Management, v. 12, n. 5, p. 431-445, 2005.

SÁ FREIRE, P.; UENO, A. T.; DIAS, M. A. H.; SANTOS, N. Ferramentas de avaliação de Gestão do Conhecimento: Um estudo bibliométrico. International Journal of Knowledge Engineering and Management (IJKEM), v. 2, n. 3, p. 15-38, 2013.

SALOJÄRVI, S.; FURU, P.; SVEIBY, K. E. Knowledge management and growth in Finnish SMEs. Journal of Knowledge Management, v. 9, n. 2, p. 103-122, 2005.

SERENKO, A.; BONTIS, N.; HULL, E. An application of the knowledge management maturity model: The case of credit unions. In: AMERICAS CONFERENCE ON INFORMATION SYSTEMS, $20^{\text {th }}$., AMCIS, 2014, Savannah, GA. Association for Information Systems.

SERNA, E. Maturity model of Knowledge Management in the interpretativist perspective. International Journal of Information Management, v. 32, n. 4, p. 365-371, Aug 2012.

Perspectivas em Gestão \& Conhecimento, João Pessoa, v. 7, Número Especial, p. 66-79, mar. 2017. 
SUJANOVA, J.; CAGANOVA, D.; SOOS, L. Innovation, knowledge and multicultural management influence on intellectual capital in industrial enterprises. Turkish Online Journal of Educational Technology, v. 2015, p. 289-300, 2015.

SUJANOVA, J.; GABRIS, P.; LICKO, M.; PAVLENDA, P.; STASIAK-BETLEJEWSKA, R. Aspects of knowledge management in Slovak industrial enterprises. In: EUROPEAN CONFERENCE ON KNOWLEDGE MANAGEMENT,13 ${ }^{\text {th }}$., ECKM, 2012, Cartagena. p.1135-1144.

WENDLER, R. The maturity of maturity model research: A systematic mapping study. Information and software technology, v. 54, n. 12, p. 1317-1339, 2012.

XIAO, J.; WANG, J.; YUE, H. Study on maturity level transition mechanism of knowledge management. In: INTERNATIONAL CONFERENCE ON INFORMATION MANAGEMENT, INNOVATION MANAGEMENT AND INDUSTRIAL ENGINEERING, ICIII 2012, 2012, Sanya. p.325328.

YANG, R.; YU, Q. Research on Knowledge Management Maturity model: Based on the life cycle of the industry. In: INTERNATIONAL CONFERENCE ON INFORMATION MANAGEMENT, INNOVATION MANAGEMENT AND INDUSTRIAL ENGINEERING, $6^{\text {th }}$., ICIII, 2013, Xi'an. p. 259-262.

Artigo recebido em 13/07/2016 e aceito para publicação em 02/03/2017

Perspectivas em Gestão \& Conhecimento, João Pessoa, v. 7, Número Especial, p. 66-79, mar. 2017. 